\title{
TBNA for the treatment of non complicated bronchogenic cyst
}

\author{
A. Andreani, G. Cappiello, M. Valli, M. Giovannini
}

A 65-year old male ex smoker came to our attention, coming from a department of General Medicine where he was admitted for a transitory ischemic attack, for the accidental detection during thoracic CT (performed as a routine chest radiograph had showed a suspect enlargement of the right pulmonary hilum) of a mediastinal round, well-circumscribed and fluid density mass adjacent to the posterior wall of the right main bronchus (fig. 1a). The patient was completely asymptomatic denying in particular fewer, cough, weight loss, retrosternal pain or dyspnea.

We, therefore, performed bronchoscopy during which we noticed an extrinsic compression of the posterior wall of the proximal part of the right main bronchus; in this site the mucosa appeared bluish and hyperemic (fig. 1b). Observing this lesion, we decided to perform a transbronchial needle aspiration (TBNA) with a collection of 35 millilitres of a viscous, brownish and turbid-type fluid (fig. 2). After the TBNA the compression of the posterior wall of the right main bronchus disappeared and the mucosa faded in colour (fig. 1c).

According to literature the patient received an antibiotic prophilaxis before and after TBNA. Cytological examination of the fluid showed a nonspecific inflammatory pattern with numerous macrophages while microbiology analysis was negative for specific pathogenic forms: these findings, supported by the radiological (CT in particular) results, were consistent with benign bronchogenic cyst.

Another thoracic CT, performed the day after, the procedure showed macroscopic reduction of the mediastinal mass (fig. 1d). Follow up examination after 3 months was almost unchanged; subsequent thoracic CT, scheduled after 4 months, will give us an indication of the evolution.

Bronchogenic cysts are lesions of congenital origin deriving from the primitive foregut and are the most common primary cysts of the mediastinum. Generally they contain clear fluid or, less commonly, hemorrhagic secretions or air. They are lined by columnar ciliated epithelium, and their walls often contain cartilage and bronchial mucous glands [1]. Although some bronchogenic cysts are asymptomatic and are incidental findings upon thoracic CT, most cysts, causing compression or irritation to the adjacent structure, could be symptomatic with cough, fever, pain, and dyspnea. Tracheobronchial compression and pulmonary infections may occur due to the relatively soft tracheobronchial tree [2]. Infection is a common complication, especially in cysts with bronchial communications.

The treatment of choice in cases presenting with complications is surgical resection with complete removal of the secreting mucosal lining. The role of surgery is however controversial in asymptomatic patients where an endoscopic aspiration could be attempted. The simple transbronchial needle aspiration (TBNA) is sometimes associated with a high recurrence rate as the lining of the cyst cannot be removed [2]. Endobronchial ultrasound transbronchial needle aspiration (EBUS-TBNA) instead, allowing visualisation during aspiration, could enable complete aspiration of the cyst causing the collapse of the cystic space with the consequent adhesion of the mucosal surfaces of the cavity; in this way one reduces the likelihood of recurrence [3].

We indeed agree with the literature regarding limiting the therapeutical use of simple TB$\mathrm{NA}$ only in cases of acute compression or in compromised or nonoperative patients [4], however, being a relatively simple and readily available technique for every bronchoscopist, in contrast to the lengthy preparation involved necessary to organize a session with EBUS, and as also suggested by some past works demonstrating a low recurrence rate [5], we think that
Monaldi Arch Chest Dis 2013; 79: 3-4, 143-145

\section{Department}

of Respiratory Diseases, St Maria Bianca Hospital, Mirandola (Modena), Italy.

Corrispondence: Alessandro Andreani $M D$, Department of Respiratory Diseases, Hospital St Maria Bianca, Mirandola (Modena), Italy; e-mail: alessandreani@yahoo.it; a.andreani@ausl.mo.it 

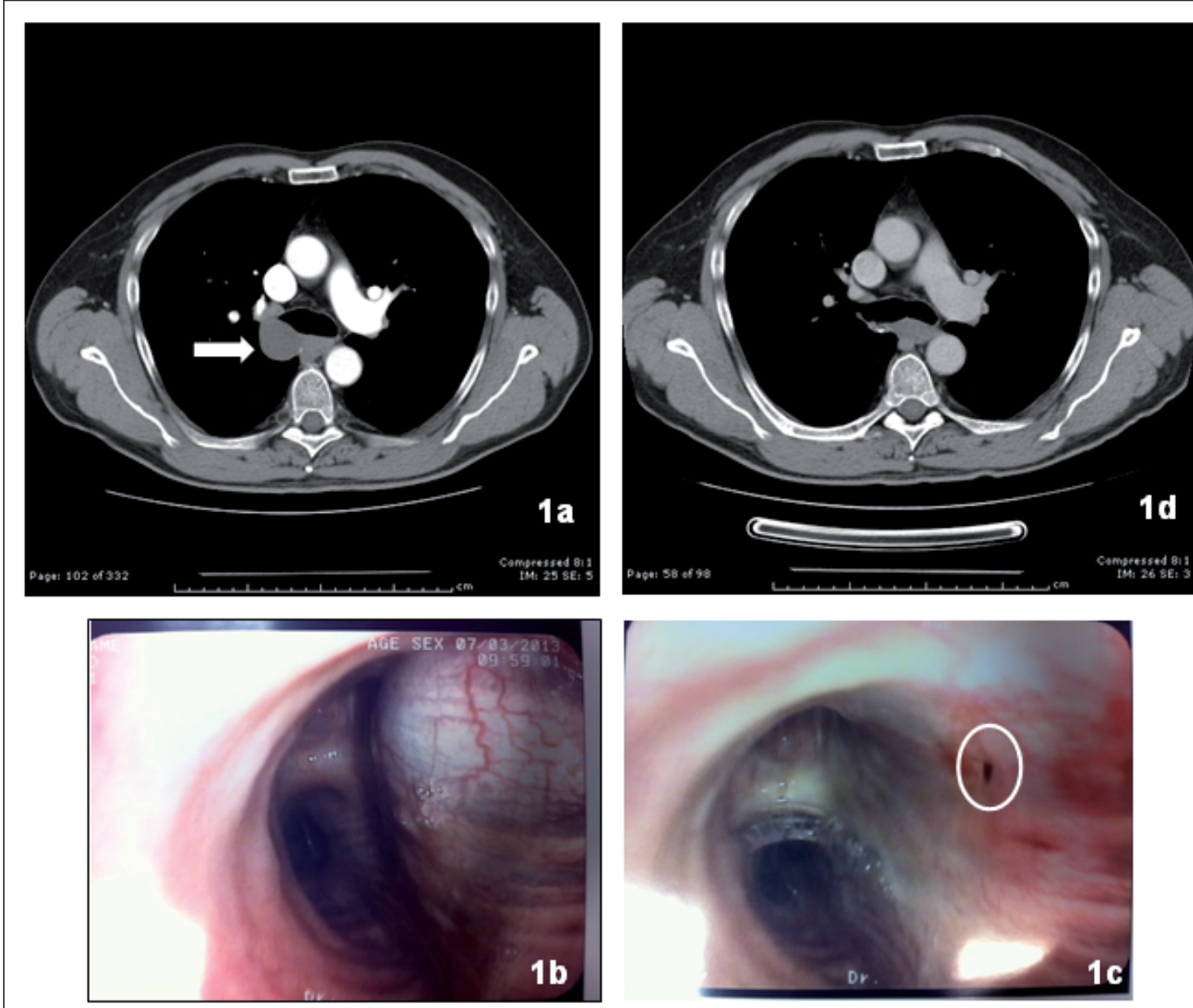

Fig. 1. - Anticlockwise: 1a CT scan showing the presence of a mediastinal round and fluid mass leaning against the posterior wal of the right main bronchus (white arrow): the well-defined margins, the absence of irregular wall, the homogeneous CT attenuation, the typical homogeneous water density (20 Hounsfield Units) and its position could already suggest a diagnosis of benign bronchogenic cyst; 1b image acquired during bronchoscopy showing the posterior wall of the beginning of the right main bronchus been tense and hyperemic for extrabronchial compression; 1c the same image as before acquired immediately after TBNA: the bronchial mucosa now appears smooth and whiter (in the white oval we can see the precise point of entry of the needle); 1d CT scan, performed after TBNA, showing a clear reduction of the size of the bronchogenic cyst. a simple TBNA could still be the most suitable technique (especially if EBUS is not readily available) as an initial approach in the management of suspected bronchogenic cysts, especially when the radiological appearance leaves no doubt regarding the benign nature of the lesion. If in fact the future follow up shows no evidence of recurrence we would avoid having the patient undergo a thoracotomy and may achieve both the diagnostic and therapeutic goal with a simpler, faster and safer technique in a single bronchoscopic session.

Clearly, if we decide to adopt a simple TBNA as the first approach treatment for suspected bronchogenic cysts, it will be necessary to program a careful clinical and radiological follow up and, therefore we must be prepared to perform safer therapeutic interventions in the event of complications or recurrence.

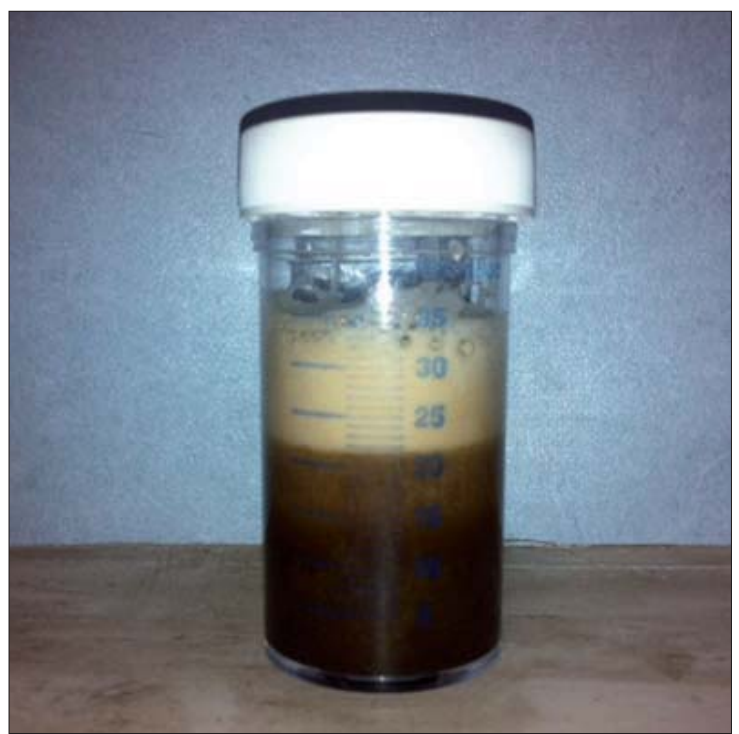

Fig. 2. - Image showing the container where we collected the brownish and torbid, but surprisingly odorless fluid taken during TBNA. As it is a very viscous fluid, we had to apply a considerable amount of suction force through the syringe connected to the needle during TBNA in order to collect as much of it as possible. 


\section{References}

1. Twehues AR, et al. Management of mediastinal bronchogenic cust with transbronchial needle aspiration. Chest 2009; 136: 23s-24s.

2. Dhand S, Krimsky W. Bronchogenic cyst treated by endobronchial ultrasound drainage. Thorax 2008; 63: 4.

3. Galluccio G, Lucantoni G. Mediastinal bronchogenic cyst's recurrence treated with EBUS-FNA with a long-term follow-up. European J of Cardio-thoracic Surgery 2006; 29: 627-629.

4. Ribet E, Copin MC, Gosselin B. Bronchogenic cyst of the mediastinum. J Thorac Cardiovasc Surg 1995; 109: 1003-10.

5. Kuhlman JE, Fishman EK, Wang KP, Zerhouni EA, et al. Mediastinal cyst: diagnosis by CT and needle aspiration. Am J Roentgenol 1988; 150: 75-8. 